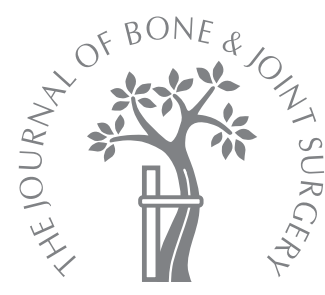

- ONCOLOGY

\title{
Early results of a non-invasive extendible prosthesis for limb-salvage surgery in children with bone tumours
}

\author{
N. Hwang, \\ R. J. Grimer, \\ S. R. Carter, \\ R. M. Tillman, \\ A. Abudu, \\ L. M. Jeys \\ From Royal \\ Orthopaedic \\ Hospital, \\ Birmingham, United \\ Kingdom
}

We reviewed our initial seven-year experience with a non-invasive extendible prosthesis in 34 children with primary bone tumours. The distal femur was replaced in $\mathbf{2 5}$ cases, total femur in five, proximal femur in one and proximal tibia in three. The mean follow-up was 44 months (15 to 86 ) and 27 patients $(79 \%)$ remain alive. The prostheses were lengthened by an electromagnetic induction mechanism in an outpatient setting and a mean extension of $32 \mathrm{~mm}$ (4 to 80 ) was achieved without anaesthesia. There were lengthening complications in two children: failed lengthening in one and the formation of scar tissue in the other. Deep infection developed in six patients $(18 \%)$ and local recurrence in three. A total of 11 patients required further surgery to the leg. Amputation was necessary in five patients $(20 \%)$ and a two-stage revision in another. There were no cases of loosening, but two patients had implant breakage and required revision. The mean Musculoskeletal Tumor Society functional score was $85 \%(60 \%$ to $100 \%)$ at last known follow-up. These early results demonstrate that the non-invasive extendible prosthesis allows successful lengthening without surgical intervention, but the high incidence of infection is a cause for concern.

Advances in chemotherapy, imaging modalities and surgical techniques have led to the increasing use of limb-salvage surgery in the treatment of primary malignant bone tumours, ${ }^{1}$ even in children, in whom the long-term survival is now over $60 \% .^{2,3}$ There is, therefore, an increasing requirement not only to preserve the limb but also maximise function and minimise complications. The maintenance of limblength equality after resection of the growth plates is also necessary.

The development of extendible endoprosthetic replacements naturally followed the initial success of fixed length prostheses and allowed the option of maintaining limb-length equality in the younger patient whose contralateral limb was still growing normally. ${ }^{4}$ The early extendible implants had a variety of designs, some modular and requiring exchange of the midsection with a longer component to achieve extension, whereas others used a worm-drive mechanism. Although early results were promising, there was a high rate of complications, including prosthesis breakage and scarring or infection from repeated operations..$^{5-8}$

In order to decrease the number of anaesthetics and operations for each lengthening, a variety of non-invasive extension mechanisms have been developed, which should decrease the risk of infection and the cost of lengthenings. ${ }^{9,10}$ Whereas these studies have generally demonstrated successful noninvasive lengthening and good functional outcomes, there have remained significant complications.

The Juvenile Tumour System (JTS; Stanmore Implants Worldwide, Stanmore, United Kingdom) was developed as another type of noninvasive extendible prosthesis and was first used in 2002. The early results have been reported. ${ }^{11,12}$ In this paper, we present our initial seven-year experience with the JTS in skeletally immature patients.

\section{Patients and Methods}

Between 2002 and 2009, 34 children underwent resection of a primary bone tumour and insertion of a non-invasive extendible prosthesis. Following institutional approval we reviewed their data on demographics, tumour characteristics, surgical complications, and functional outcome.

There were 18 boys and 16 girls with a mean age of 11 years (7 to 14), of whom 31 had an osteosarcoma and three a Ewing's sarcoma. The distal femur was replaced in 25 patients, total femur in five, proximal femur in one and the proximal tibia in three. All the patients had chemotherapy according to the current international trial protocols (EURAMOS ${ }^{13}$ and EuroEWING, ${ }^{14}$ respectively) which involved 


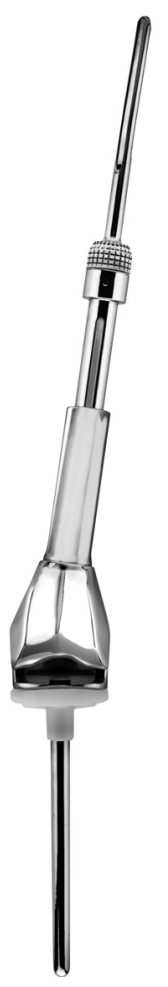

Fig. 1

Photograph showing the custom designed non-invasive extendible prosthesis based on planned tumour resection levels. The collar is coated with hydroxyapatite.

neoadjuvant and adjuvant chemotherapy. One patient with Ewing's sarcoma had pre-operative radiotherapy.

Six-weeks post-operatively, all patients underwent a standardised one-week regime of intensive inpatient physiotherapy to maximise their recovery. They were followed up regularly and offered limb lengthening when there was an obvious discrepancy, usually about $1 \mathrm{~cm}$. Functional outcome was assessed by the Musculoskeletal Tumor Society Score (MSTS) ${ }^{15}$ at the last follow-up. The survival of the implant was calculated using the Kaplan-Meier method.

The Stanmore non-invasive extendible prosthesis has been well described (JTS; Stanmore Implants Worldwide Ltd). ${ }^{16}$ The custom-designed implant lengthens non-invasively by the principles of electromagnetic induction. The shaft of the prosthesis is constructed in two segments, which slide longitudinally during the lengthening process. The inner shaft telescopes via a power screw driven by an epicyclic gearbox, whereby one turn of the screw extends the shaft by $1 \mathrm{~mm}$. The input shaft of the gearbox is connected to a magnetic disc made of rare earth $\mathrm{NdFeB}$ metal and the gearbox produces an overall speed reduction of 13061:1 and an output torque of $4 \mathrm{Nm}$ to drive the screw. The use of MRI in the follow-up of these patients is contraindicated due to magnetic interference with the electromagnetic motor and potential damage to the MR scanner. The implant has to be sterilised

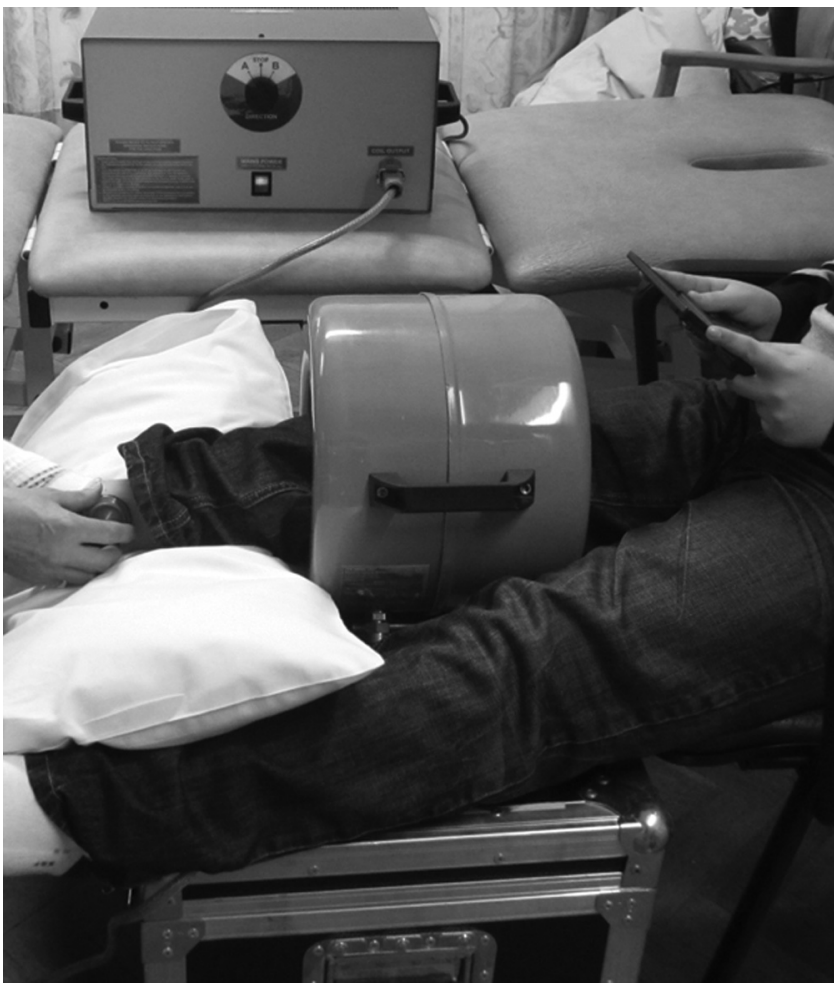

Fig. 2

Photograph showing the lengthening procedure. The operated limb is placed inside an external portable electromagnetic circular drive unit. When activated, a rotating magnetic field is induced and the drive unit captures the implanted magnetic disc, causing it to rotate synchronously. The child is undergoing lengthening of a distal femoral replacement. The procedure is totally pain-free. The physiotherapist can listen to the leg to hear if the motor is working.

with gamma irradiation and cannot be autoclaved as this damages the magnet. The extending mechanism of the implant comes in three sizes, allowing $50 \mathrm{~mm}, 70 \mathrm{~mm}$ or 90 $\mathrm{mm}$ of extension and these are attached to the stem and joint of the implant (Fig. 1).

The prosthesis is lengthened by placing the operated limb inside an external electromagnetic circular drive unit (Fig. 2). This unit is controlled by a single-phase $220 \mathrm{~V}$ power unit. Both units are portable, with the drive and power units weighing $32 \mathrm{~kg}$ and $16 \mathrm{~kg}$ respectively. When activated, a rotating magnetic field at a speed of $3000 \mathrm{rpm}$ is induced and the drive unit captures the implant magnetic disc causing it to rotate synchronously. The implant extends at a constant rate of $0.23 \mathrm{~mm}$ per minute at full speed. This process enables the growing prosthesis to be extended under axial loads of up to $1350 \mathrm{~N}$, which is enough to overcome increasing soft-tissue tension during lengthening. ${ }^{17}$ In order to prevent overloading the prosthesis, each session is typically performed over 20 minutes, producing $5 \mathrm{~mm}$ of extension. In selected sessions, repeated consecutive application of the drive unit at a boosted voltage was required in order to achieve extension for a few patients. Active physiotherapy was also regularly given 


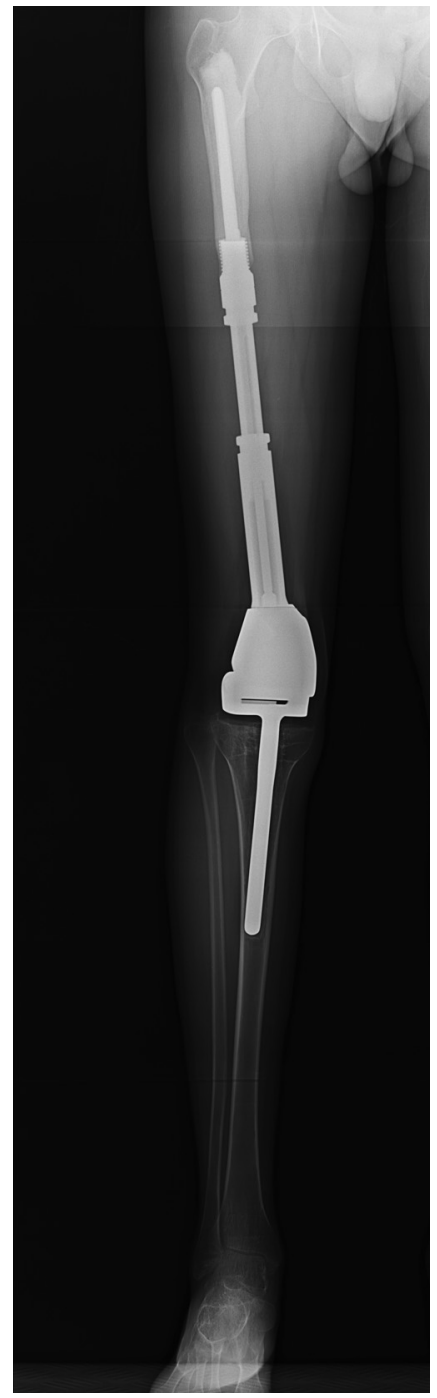

Fig. 3

Radiograph showing the non-invasive prosthesis of the right distal femur following $60 \mathrm{~mm}$ of lengthening. The functional score was $95 \%$. Note the bony ingrowth into the hydroxyapatite collar at the bone-prosthesis junction.

between sessions to maximise joint movement and reduce surrounding soft-tissue resistance for subsequent lengthenings.

Patients were selected for a non-invasive lengthening prosthesis if they were expected to have a minimum of $30 \mathrm{~mm}$ of growth remaining in the resected epiphysis and if the length of bone being resected allowed insertion of the prosthesis. The first lengthening was not usually undertaken until well after the patient had completed adjuvant chemotherapy and was usually when they or their parents began to notice a limb-length discrepancy. Routine radiographs were performed after the initial lengthening procedure to confirm its success. None of the lengthenings required a general anaesthetic and all were performed on an outpatient basis. Whereas the procedure was universally reported to be painless, some patients described a sensation of stretching during lengthening.

Operative technique. All primary resections and prosthetic replacements were conducted by one of the four senior authors. The operative technique has been previously described. ${ }^{18}$ All of the implants were custom designed and cemented into the intramedullary cavity to achieve stable fixation, with the collar of the prosthesis coated with hydroxyapatite. ${ }^{19}$ In all cases the bone on the opposite side of the joint was reconstructed using a 'sliding' component, which crossed the growth plate but allowed continued growth of the epiphysis. ${ }^{20}$ Prophylactic antibiotics were given to all patients using cefuroxime at the time of induction of anaesthesia and for up to 24 hours post-operatively. Palacos G cement (Heraeus Kulzer GmbH, Hanau, Germany) containing gentamicin was used for the cemented components. All the children undergoing proximal tibial replacement had an associated gastrocnemius muscle flap reconstruction.

\section{Results}

A total of 19 patients $(56 \%)$ had a good response to chemotherapy with $>90 \%$ necrosis. Three $(9 \%)$ developed local recurrence, two of whom were already known to have metastases and the other developed metastases three months later. All three required amputation. A total of 13 patients $(38 \%)$ developed metastases, of whom seven died after a mean of 23 months (4 to 42). A total of 27 patients $(79 \%)$ remain alive at a mean follow-up of 44 months (15 to 86 ) from the date of surgery.

The mean extension designed into the prosthesis was $60 \mathrm{~mm}$ (50 to 90 ) and 27 patients $(79 \%$ ) have undergone lengthening. The first was carried out when a mean leglength discrepancy of $16 \mathrm{~mm}$ (5 to 40) had developed. This was at a mean of 12.5 months (3 to 33 ) from the original operation. A mean lengthening of $4 \mathrm{~mm}$ was achieved at each lengthening session, with a mean of five sessions (1 to $15)$ for each patient. The mean total lengthening to date is $32 \mathrm{~mm}$ (4 to 80) and an example of $60 \mathrm{~mm}$ lengthening is shown in Figure 3. In the seven patients who have not undergone a lengthening, one died from metastases after four months, two developed an infection and one had a local recurrence leading to amputation. In three cases with shorter follow-up, no limb-length discrepancy is yet apparent.

In four patients a booster voltage from $120 \mathrm{~V}$ to $170 \mathrm{~V}$ was necessary to attain the required extension. There were complications of the lengthening device in two patients, with failed lengthening in one (the implant was inadvertently sterilised) and scar tissue formation in the other. One patient required revision to a second lengthening prosthesis, having reached the $70 \mathrm{~mm}$ maximum lengthening capacity of the first.

Complications. Six patients $(18 \%)$ developed deep infection at a mean of five months $(0$ to 15$)$ from the initial operation (Table I). Another developed a superficial wound infection, which settled with antibiotics alone. 
Table I. Details of the six cases with deep infection showing the age of the patients, site of the prosthesis, time in months to infection and its cause, management and outcome

\begin{tabular}{|c|c|c|c|c|c|c|c|}
\hline Patient & Age (yrs) & Site ${ }^{*}$ & $\begin{array}{l}\text { Time to } \\
\text { infection } \\
\text { (mths) }\end{array}$ & Cause & Organism $^{\dagger}$ & Management & Outcome \\
\hline 1 & 11 & DF & 0 & Wound necrosis & CNS and pseudomonas & $\begin{array}{l}\text { Washout, antibiotics, } \\
\text { local flap }\end{array}$ & Resolved \\
\hline 2 & 10 & DF & 1 & $\begin{array}{l}\text { Neutropaenic sepsis during } \\
\text { chemotherapy }\end{array}$ & CNS & Antibiotics & Resolved \\
\hline 3 & 12 & PT & 2 & Wound necrosis & CNS and enterococci & Two stage revision & Amputation \\
\hline 4 & 11 & DF & 15 & Central line infection & CNS & Washout and antibiotics & $\begin{array}{l}\text { Resolved, amputation } \\
\text { for local recurrence }\end{array}$ \\
\hline 5 & 14 & TF & 8 & $\begin{array}{l}\text { Wound necrosis after pre- } \\
\text { operative radiotherapy }\end{array}$ & Staphylococcus aureus & $\begin{array}{l}\text { Debridement, free flap, } \\
\text { antibiotics }\end{array}$ & Amputation \\
\hline 6 & 13 & DF & 6 & Central line infection & Streptococcus viridans & Washout and antibiotics & $\begin{array}{l}\text { Concurrent local recur- } \\
\text { rence, amputation }\end{array}$ \\
\hline
\end{tabular}

* DF, distal femur; PT, proximal tibia; TF, total femur

† CNS, coagulase-negative staphylococcus

There were no cases of loosening. Two patients underwent revision for implant breakage, one for the sliding component and one for the lengthening part of the prosthesis after $47 \mathrm{~mm}$ of extension.

Two patients had a partial sciatic nerve palsy. One resolved completely but the other was left with sensory changes in the foot.

The mean MSTS score at the most recent follow-up was $85 \%(60 \%$ to $100 \%)$. Seven children developed a fixed flexion deformity. Three resolved with physiotherapy, three required a manipulation under anaesthesia and one required excision of scar tissue.

Overall, further surgery to the leg was needed in 11 patients (32\%), five for infection, three for failure of the implant and five for amputation (three for local recurrence and two for infection). The survival of the implant without revision or amputation following primary surgery was $77 \%$ at three years and $52 \%$ at five years.

\section{Discussion}

There is considerable experience with the use of invasive lengthening prostheses and it is generally accepted that the main drawbacks are the high incidence of complications and repeated general anaesthetics for each lengthening, leading to a significant risk of further complications, including infection. ${ }^{22}$ It has been suggested that each open lengthening leads to a $2 \%$ to $5 \%$ risk of deep infection ${ }^{7}$ and therefore, non-invasive prostheses have become more popular.

Early experience with non-invasive extendible prostheses was described by Wilkins and Soubeiran, ${ }^{23}$ using a compressed spring within a titanium tube which was locked to an outer polyethylene cylinder. Under fluoroscopic guidance, extension was achieved by external electromagnetic induction which selectively heated the polyethylene cylinder above $130^{\circ} \mathrm{C}$ and allowed a controlled release of the spring's stored energy to lengthen the prosthesis. Whereas early results were promising, longer term follow-up showed a high rate of failure. ${ }^{9,10}$
Early results of the Stanmore non-invasive extendible prostheses were reported by Gupta et al. ${ }^{11}$ In their series of seven children, the surgical and oncological outcomes were encouraging with no reported mechanical difficulties and no cases of infection, although the mean MSTS score was only $68 \%$ at a mean follow-up of 20.2 months (14 to 30 ).

Our results are still relatively short-term but show the high incidence of complications with this type of surgery in the growing child. ${ }^{22}$ Of particular concern is the incidence of infection in this cohort, at $18 \%$. Most of these infections arose early and were related either to problems with wound healing or complications of chemotherapy. In this group of patients, meticulous peri-operative wound care is essential and any areas that fail to heal should be excised and if necessary reconstructed with appropriate flaps at an early stage to prevent infection. In one patient the infection arose after pre-operative radiotherapy, which is a known risk factor for infection after limb salvage surgery. ${ }^{24}$ The development of peri-prosthetic infection during chemotherapy is also a well recognised complication and two patients developed central line infections, which had spread to the implants shortly after they had completed their chemotherapy. Careful attention to asepsis in patients with central lines in situ is essential at all times but especially in those with an endoprosthesis. Early removal of the line once chemotherapy is completed is recommended.

The possible role of silver, either as a coating or as a surface modification to reduce the risk of infection, is currently being investigated in a number of centres, including our own, but it is premature to say whether this will help. ${ }^{25,26}$

It is possible that the ability to insert a non-invasive extendible prosthesis has tempted us to undertake limb salvage, whereas amputation would have been advised previously. This is likely to have been the case in two of the three children with wound necrosis and subsequent infection. In one, all the vasti were removed, leaving just rectus femoris to cover the prosthesis anteriorly and, in another, the preoperative radiotherapy compromised wound healing. In both cases, amputation might have been preferable. Other 
alternatives such as rotationplasty or primary amputation should also be considered, especially if the soft tissues left covering the prosthesis are likely to be inadequate. ${ }^{27}$

The management of an infected endoprosthesis is similar, regardless of the age of the patient. ${ }^{28}$ However, in a child undergoing chemotherapy, the treatment of the infection may be compromised by the treatment of the tumour. The combination of local recurrence and infection is unusual but led to the need for amputation in two of our patients.

Mechanical problems are always a potential risk with any implant and children who have a successful outcome from limb salvage are likely to use their implants to the full. This may account for the two fractures in this series, both in very active patients with functional scores over $90 \%$. The mean MSTS score of $85 \%$ at 31 months was comparable with other studies and better than the $68 \%$ reported by Gupta et al. ${ }^{11}$

We considered the possibility that either the prosthesis or its magnetic lengthening system could in some way have contributed to the development of local recurrence or infection. In such a small series, it is impossible to confirm this and there is no scientific reason why this should be the case. However, as with any new implantable device, it is a factor that should be monitored. The size of the motor is greater than either a minimally invasive or non-extending prosthesis and it is possible that the increased bulk, particularly in very young children, may have contributed to problems with wound healing.

Our results confirm that the JTS can achieve its aim of allowing repeated lengthening without operative intervention. However, the results are marred by the worryingly high rate of infection, which needs to be considered in each case. The additional cost of the JTS over an older, minimally invasive prosthesis needs to be justified by taking into account the overall cost of the child's treatment until maturity, including the costs of lengthenings and treatment of any complications. This will be the subject of a more detailed future study.

No benefits in any form have been received or will be received from a commercial party related directly or indirectly to the subject of this article.

\section{References}

1. Wafa H, Grimer RJ. Surgical options and outcomes in bone sarcoma. Expert Rev Anticancer Ther 2006;6:239-248.

2. Wilkins R, Cullen J, Odom L, et al. Superior survival in treatment of primary nonmetastatic pediatric osteosarcoma of the extremity. Ann Surg Oncol 2003;10:498507.

3. Paulussen M, Craft AW, Lewis I, et al. Results of the EICESS-92 Study: two randomized trials of Ewing's sarcoma treatment: cyclophosphamide compared with ifosfamide in standard-risk patients and assessment of benefit of etoposide added to standard treatment in high-risk patients. J Clin Oncol 2008;26:4385-4393.
4. Baumgart R, Betz A, Schweiberer L. A fully implantable motorized intramedullary nail for limb lengthening and bone transport. Clin Orthop 1997;343:135-143.

5. Unwin PS, Walker PS. Extendible endoprostheses for the skeletally immature. Clin Orthop 1996;322:179-193.

6. Lewis MM. The use of an expandable and adjustable prosthesis in the treatment of childhood malignant bone tumors of the extremity. Cancer 1986;57:499-502.

7. Abudu A, Grimer R, Tillman R, Carter $\mathbf{S}$. The use of prostheses in skeletally immature patients. Orthop Clin North Am 2006;37:75-84.

8. Schiller C, Windhager R, Fellinger EJ, et al. Extendible tumour endoprostheses for the leg in children. J Bone Joint Surg [Br] 1995;77-B:608-614.

9. Neel MD, Wilkins RM, Rao BN, Kelly CM. Early multicenter experience with a noninvasive expandable prosthesis. Clin Orthop 2003;415:72-81.

10. Saghieh S, Abboud MR, Muwakkit SA, et al. Seven-year experience of using Repiphysis expandable prosthesis in children with bone tumors. Pediatr Blood Cancer 2010;55:457-463.

11. Gupta A, Meswania J, Pollock R, et al. Non-invasive distal femoral expandable endoprosthesis for limb-salvage surgery in paediatric tumours. J Bone Joint Surg [Br] 2006;88-B:649-654.

12. Sewell MD, Spiegelberg BG, Hanna SA, et al. Non-invasive extendible endoprostheses for limb reconstruction in skeletally-mature patients. J Bone Joint Surg [Br] 2009;91-B:1360-1365.

13. Marina N, Bielack S, Whelan J, et al. International collaboration is feasible in trials for rare conditions: the EURAMOS experience. Cancer Treat Res 2010;152:339353.

14. Juergens C, Weston C, Lewis I, et al. Safety assessment of intensive induction with vincristine, ifosfamide, doxorubicin and etoposide (VIDE) in the treatment of Ewing tumours in the EURO-E.W.I.N.G. 99 clinical trial. Pediatr Blood Cancer 2006; $47: 22-29$.

15. Enneking WF, Dunham W, Gebhardt MC, Malawar M, Pritchard DJ. A system for the functional evaluation of reconstructive procedures after surgical treatment of tumors of the musculoskeletal system. Clin Orthop 1993;286:241-246.

16. Meswania JM, Taylor SJ, Blunn GW. Design and characterization of a novel permanent magnet synchronous motor used in a growing prosthesis for young patients with bone cancer. Proc Inst Mech Eng 2008;222:393-402.

17. Meswania JM, Walker PS, Sneath RS, Grimer RJ. In vivo distraction forces in extendible endoprosthetic replacements: a study of 34 patients. Proc Inst Mech Eng H 1988;212:151-155

18. Malawer MM. Distal femoral resection for sarcomas of bone. In: Sugarbaker PH, Malawer MM, eds. Musculoskeletal surgery for cancer. New York: Thieme Medical Publishers, 1992:243-259.

19. Myers G, Abudu A, Carter S, Tillman R, Grimer R. Endoprosthetic replacement of the distal femur for bone tumours: long-term results. J Bone Joint Surg [Br] 2007;89B:521-526

20. Cool WP, Carter SR, Grimer RJ, Tillman RM, Walker PS. Growth after extendible endoprosthetic replacement of the distal femur. J Bone Joint Surg [Br] 1997;79B:938-942.

21. Grimer RJ, Carter SR, Tillman RM, et al. Endoprosthetic replacement of the proximal tibia. J Bone Joint Surg [Br] 1999;81-B:488-494.

22. Jeys $\mathbf{L}$, Grimer $\mathbf{R}$. The long-term risks of infection and amputation with limb salvage surgery using endoprostheses. Recent Results Cancer Res 2009;179:75-84.

23. Wilkins RM, Soubeiran A. The Phenix expandable prosthesis: early American experience. Clin Orthop 2001;382:51-58.

24. Jeys LM, Luscombe JS, Grimer RJ, et al. The risks and benefits of radiotherapy with massive endoprosthetic replacement. J Bone Joint Surg [Br] 2007;89-B:13521355.

25. Gosheger G, Hardes J, Ahrens H, et al. Silver-coated megaendoprostheses in a rabbit model: an analysis of the infection rate and toxicological side effects. Biomaterials 2004;25:5547-5556.

26. Hardes J, Ahrens $\mathbf{H}$, Gebert C, et al. Lack of toxicological side-effects in silvercoated megaprostheses in humans. Biomaterials 2007;28:2869-2875.

27. Veenstra KM, Sprangers MA, van der Eyken JW, Taminiau AH. Quality of life in survivors with a Van Ness-Borggreve rotationplasty after bone tumour resection. J Surg Oncol 2000;73:192-197.

28. Leone JM, Hanssen AD. Management of infection at the site of a total knee arthroplasty. J Bone Joint Surg [Am]2005;87-A:2335-2348. 